\title{
Phenotypic clusters and survival analyses in interstitial pneumonia with myositis-specific autoantibodies
}

Yihua Li

Beijing Chaoyang Hospital

Shiwen Yu

Beijing Chaoyang Hospital

Yali Fan

Beijing Chaoyang Hospital

$\mathrm{Na} \mathrm{Wu}$

Beijing Chaoyang Hospital

Yiran Wang

Beijing Chaoyang Hospital

Qiao Ye ( $\nabla$ yeqiao_chaoyang@sina.com )

Beijing Chaoyang Hospital, Capital Medical University https://orcid.org/0000-0002-0932-0487

Research article

Keywords: autoantibody, cluster analysis, interstitial pneumonia, myositis, prognosis

Posted Date: September 22nd, 2020

DOI: https://doi.org/10.21203/rs.3.rs-38365/v2

License: (c) (1) This work is licensed under a Creative Commons Attribution 4.0 International License.

Read Full License 


\section{Abstract}

Background Interstitial pneumonia (IP) is one of the common pulmonary complications of idiopathic inflammatory myopathy (IIM), among which myositis-specific autoantibodies (MSAs) are specific for diagnosing and predicting the prognosis of IIM. However, IP patients with MSAs (MSA-IP) have not been well described. The study aimed to explore the phenotypic clusters and prognosis of MSA-IP patients. Methods A total of 124 MSA-IP patients were prospectively enrolled for analysis. Serum MSAs were detected using immunoprecipitation. Radiographic patterns of IP were determined according to the classification of idiopathic IPs. Clusters of MSA-IP patients were identified using cluster analysis.

Potential risk factors for acute-onset disease and short-term prognoses were also analysed. Results There were four clusters of MSA-IP patients. Cluster 1 patients were elderly with chronic onset and a usual interstitial pneumonia pattern on computed tomography. Cluster 2 patients were all positive for antiaminoacyl-tRNA antibodies, were predominantly female and had frequent respiratory symptoms. Patients in cluster 3 showed multi-system involvement with a nonspecific interstitial pneumonia pattern. Patients in cluster 4 had severe respiratory symptoms with anti-MDA5 antibodies. The patients in cluster 3 (OR $6.682,95 \% \mathrm{Cl} 1.560-28.622, \mathrm{P}=0.011$ ) and cluster 4 (OR 6.057, 95\% $\mathrm{Cl} 1.715-21.388, \mathrm{P}=0.005$ ) were susceptible to acute-onset disease. The patients in cluster 4 were prone to disease progression (HR 2.711, $95 \% \mathrm{Cl} 1.128-6.519, \mathrm{P}=0.034)$, which was consistent with the Kaplan-Meier curves. Conclusions Four distinctive clusters were determined by cluster analysis, suggesting the characteristics, serological antibodies and prognosis of patients with MSA-IP.

\section{Background}

Interstitial lung diseases (ILDs) are a heterogeneous group of diffuse and fibrotic pulmonary disorders with extensive morbidity and mortality, characterized by the loss of alveolar-capillary functional structures [1]. ILDs are generally classified into four subgroups: diseases with known causes such as occupational and environmental exposure, connective tissue diseases (CTDs) and drug-related ILDs; idiopathic interstitial pneumonias (IIPs); granulomatous lung disorders such as sarcoidosis; and specific entities [2]. The underlying causes of some ILDs are still unknown, leading to challenges in early diagnosis and treatment $[1,3]$.

Interstitial pneumonia (IP) is a well-acknowledged manifestation of CTD. IP occurring within the context of CTD is referred to as CTD-associated IP [4,5]. A study summarized the incidence of CTDs combined with ILD, including systemic sclerosis, rheumatoid arthritis, Sjogren's syndrome, mixed CTD, polymyositis (PM), dermatomyositis (DM), and systemic lupus erythema, and finally calculated that the incidence of CTD-associated ILD (CTD-ILD) is 15\% [6]. IP can be the primary or sole manifestation of CTD [7], leading to difficulties in obtaiing an accurate diagnosis at the first clinical visit. When patients with IP have clinical, serological, and/or morphological features likely stemming from underlying autoimmune conditions but do not satisfy the diagnostic criteria for any CTD, they are diagnosed with IP with autoimmune features (IPAF) [5]. 
Idiopathic inflammatory myopathies (IIMs) are one of the unusual subtypes of CTDs. IIMs are characterized by skeletal muscle inflammation and include PM, DM, amyopathic dermatomyositis (ADM), inclusion body myositis, etc. [8,9]. IP is one of the most common extra-muscular manifestations of IIM. [10] The prevalence of IIM-associated ILD is reported to range from $19.9 \%$ to $86 \%$ [11-17]. The autoantibodies of IIMs consist of myositis-specific autoantibodies (MSAs) and myositis-associated autoantibodies (MAAs). MSAs are highly specific and include anti-aminoacyl-tRNA-synthetase antibodies (anti-ARS), anti-Mi-2 antibodies, anti-MDA5 antibodies and so on, whereas MAAs are less specific and can be detected in other CTDs [18]. MSAs are essential for assessing the clinical characteristics, diagnosis and prognosis of IIMs [19].

Previous studies have mainly focused on PM/DM secondary to ILDs [20]. However, the clinical characteristics and prognosis of IP with positive MSA expression (MSA-IP), irrespective of whether IIM has been diagnosed, are vague. Cluster analysis is an effective method for identifying homogeneous phenotypes among patients with heterogeneous disorders $[21,22]$. Therefore, this study aimed to explore the clinical characteristics and potential risk factors for the acute onset and progression of MSA-IP patients using cluster analysis.

\section{Methods}

Study cohort

A total of 2,115 patients with IP from Beijing Chao-Yang Hospital were sequentially and prospectively included from January 2017 to September 2019. IP was diagnosed according to the 2013 American Thoracic Society (ATS) and European Respiratory Society (ERS) Consensus Classification of IIPs [23]. All patients with IP underwent clinical examinations, laboratory tests, high-resolution computed tomography (HRCT) of the chest, pulmonary function tests, and, if necessary, pathological examinations at their first clinical visits [23].

Among the enrolled patients, 30 patients were diagnosed with PM, 20 with DM, 33 with ADM and 41 with IPAF. IIM was diagnosed according to the European League Against Rheumatism (EULAR)/American College of Rheumatology (ACR) criteria for definite IIM, and subclassification was performed using the classification tree in the criteria [24]. IPAF was diagnosed using the ERS/ATS research statement [5]. Of the 2,115 patients with IP, 42 underwent pathological examinations of the lungs and no patients received a lung transplant.

\section{Data collection and definitions}

At the first clinical visit, the patient medical records were reviewed to uniformly extract clinical data, including demographics (age, sex, and smoking status), patient-reported information (date of IP-related 
symptom onset, including cough and dyspnoea), clinical manifestations, physical examinations and comorbidities.

Serological markers were obtained within one month of presentation to the clinic, including C-reactive protein, erythrocyte sedimentation rate, fibrinogen, immunoglobulin (Ig) A, IgG and IgM. Levels of autoantibodies, creatine kinase and cardiac troponin I were also recorded. MSAs, including anti-ARS (antiJo-1, anti-PL-7, anti-PL-12, anti-OJ, and anti-EJ), anti-SRP, anti-Mi-2, anti-MDA5, anti-TIF1Y, anti-NXP2, and anti-SAE antibodies were detected by immunoprecipitation, as previously reported [25-27].

IP was diagnosed by HRCT. All enrolled patients underwent chest HRCT with a 1-s scan time, $0.625-\mathrm{mm}$ sections, and 10-mm intervals from the lung apex to the base including both lungs in the field of view. Each HRCT scan was reviewed independently by two experienced thoracic radiologists blinded to the clinical data. HRCT patterns were classified as usual interstitial pneumonia (UIP), nonspecific interstitial pneumonia (NSIP), organic pneumonia, or diffuse ground-glass opacity (GGO) according to the classification of IIPs [23]. The interobserver correlation was good. The kappa value was 0.83 .

A pulmonary function test was performed for each patient. The test items included forced vital capacity (FVC) and the diffusing capacity of the lung for carbon monoxide (DLCO) using the single-breath method [28].

Smoking status was categorized into non-smokers, ex-smokers (quit smoking $\geq 12$ months previously) and current smokers (currently smoking or quit smoking $<12$ months previously). Acute (or subacute) onset was defined as less than three months from symptom onset to the first clinical visit, and chronic onset was defined as a duration of more than three months. Malignancy was recorded if it occurred within three years before or after a positive detection of MSAs [29]. Pulmonary hypertension was considered if the tricuspid regurgitation velocity was $\leq 2.8 \mathrm{~m} / \mathrm{s}$ and/or the systolic pulmonary arterial pressure was $\geq 37 \mathrm{mmHg}$ on echocardiography [30].

Follow-up and endpoint of the study

The outcomes of this study were the progression of IP defined as a relative decrease of FVC\% predicted $\geq 10 \%$, a relative decrease of DLCO $\%$ predicted $\geq 15 \%$, or death within 6 months of the IP diagnosis [31]. The follow-up interval was 3 or 6 months, and the follow-up period ended in September 2019. Survival time was calculated from the onset of IP-related symptoms to the outcome or end of the follow-up period.

Statistical analysis

Quantitative data are reported as the means \pm standard deviations or medians (interquartile ranges), and qualitative data are reported as numbers and percentages. With the TwoStep Cluster algorithm, the 
clustering criterion was the Bayesian Information Criterion, the distance measurement form was logarithmic likelihood, the number of clusters was automatically determined by the algorithm, and the maximum value was set as 15 clusters. The variables included in the cluster analysis were all categorical variables related to the patients' clinical characteristics, myositis autoantibodies and imaging findings. The variables included pulmonary symptoms (including cough and dyspnoea), skeletal muscle symptoms (including proximal muscle weakness and dysphagia), UIP patterns, and MSA subtypes (antiARS, anti-SRP, anti-Mi2, anti-TIF1Y, anti-MDA5, anti-NXP2, and anti-SAE). These variables were available for all participants. Analysis of variance was used for comparisons of normally distributed quantitative data, the non-parametric Mann-Whitney $U$ test was used to compare quantitative data with a non-normal distribution, and the chi-square test was used for comparisons of qualitative data. Multivariable logistic regression was applied to determine potential risk factors for acute-onset disease. Survival curves were obtained using Kaplan-Meier curves and compared with log-rank tests. A multivariable Cox proportional hazards model was constructed to identify prognostic factors. Statistical analysis was performed using SPSS software (version 23.0, IBM), and $P<0.05$ was statistically significant.

\section{Results}

Demographics

A total of 124 patients with MSA-IP were enrolled in cluster analysis after evaluation (Additional file1: Figure S1) and were categorized into four clusters (Figure 1). As shown in Additional file 2: Table S1, the mean age of the study population was $57.5 \pm 11.2$ years, and $58.1 \%$ were females. Chronic-onset diesase (57.3\%) was common. Cluster 1 had $25(20.2 \%)$ patients with a mean age of $60.8 \pm 12.3$ years, and $80 \%$ had chronic-onset. Cluster 2 was the largest cluster (54/124, 43.6\%), with the highest proportion of females $(74.1 \%, P=0.008)$. Cluster 3 comprised 15 patients, and acute-onset disease was common $(60.0 \%, P=0.023)$. Cluster 4 had the smallest proportion of females $(36.7 \%, P=0.008)$ and a high proportion of patients with acute-onset disease (56.7\%).

\section{Clinical characteristics}

In the study population, pulmonary involvement was the most common (Table 1). Cough and dyspnoea were frequently observed in cluster $1(88 \%, 68 \%)$ and cluster $2(88.9 \%, 88.9 \%)$. In cluster 3 , proximal muscle weakness $(66.7 \%, P<0.001)$, morning stiffness $(26.7 \%, P=0.036)$, joint swelling $(40 \%, P=0.001)$ and cutaneous involvement were present (Table 1 and Additional file 3: Table S2). Patients in cluster 4 were characterized by fever $(56.7 \%, P=0.033)$ and cough $(93.3 \%, P<0.001)$.

MSA and laboratory measurements 
Among the 124 patients with MSA-IP, $60.5 \%$ were positive for anti-ARS antibodies, of which anti-Jo-1 and anti-PL-7 antibodies were the most frequent subtypes, and anti-MDA5 antibodies were the most common subtype of anti-non-ARS MSA (Table 2). All cluster 2 patients were positive for anti-ARS antibodies, in which anti-Jo-1 $(24.1 \%, P=0.012)$ and anti-EJ $(31.5 \%, P<0.001)$ antibodies were representative. The composite physiologic index was the highest in cluster $2(43.2 \pm 12.2, P=0.025)$. In cluster 3 , anti-PL-7 $(26.7 \%, P=0.010)$ antibodies were common, and cardiac troponin I was elevated $[0.03(0.02,0.06) \mathrm{ng} / \mathrm{ml}$, $P=0.019$ ] (Table 2). The distinctive MSA subtypes of cluster 4 were anti-MDA5 $(36.7 \%, P<0.001)$ and anti-Mi-2 $\beta(26.7 \%, P<0.001)$ antibodies. Supplementary laboratory tests are shown in Additional file 4: Table S3.

\section{HRCT findings}

Among these 124 patients, the most frequent HRCT pattern was NSIP (34.7\%), followed by UIP $(20.2 \%)$ (Additional file 5: Table S4). Cluster 1 patients all had UIP patterns, and NSIP patterns were frequent in cluster $3(53.3 \%, P<0.001)$. In cluster 4 , diffuse GGOs $(23.3 \%, P[0.001)$ were representative.

Risk factors for acute onset

After adjusting for age, sex, and smoking status, the patients in cluster 3 (OR 6.682, 95\% Cl 1.56028.622, $P=0.011$ ) and cluster 4 (OR 6.057, 95\% $\mathrm{Cl} 1.715-21.388, P=0.005)$ were more likely to have acuteonset disease by multivariable logistic regression analysis (Table 3 ).

\section{Survival}

The outcomes and median survival time of the four clusters are shown in Additional file 6: Table S5. The Kaplan-Meier curves showed that the prognosis of cluster 4 was the worst among all clusters $\left(c^{2}=9.138\right.$, log rank $P=0.028$ ) (Figure 2). The median survival time of cluster 4 was also the shortest. The patients in cluster 4 were prone to disease progression (HR 2.711, 95\% $\mathrm{Cl} 1.128-6.519, P=0.026$ ) after adjusting for age, sex and smoking status by a multivariable Cox proportional hazards model, which was in line with the Kaplan-Meier curves (Table 4).

\section{Discussion}

To the best of our knowledge, the present study is the first report to use cluster analysis to classify MSAIP patients into four distinctive clusters: cluster 1, elders with a UIP pattern; cluster 2, patients with pulmonary symptoms and anti-ARS antibodies; cluster 3, patients with multi-system involvement, acute onset, and an NSIP pattern; and cluster 4, patients with acute onset, disease progression and anti-MDA5 
antibodies. The classification of patients was based on clinical features, autoantibodies and prognosis. The clusters may assist clinicians in identifying and assessing the risk of disease progression in patients with MSA-IP before the IIM subtype is diagnosed.

Previous studies have indicated the potential importance of MSAs in diagnosing and predicting the prognosis of IP. A cohort study showed that $26.7 \%$ (44/165) of patients with IP at the initial diagnosis were positive for myositis autoantibodies [32]. In addition, the overall survival rate of patients with IP and anti-ARS antibodies was higher than that of patients with idiopathic pulmonary fibrosis (IPF), and the survival rate of IP with anti-ARS antibodies was similar regardless of whether IIM was diagnosed [20]. These data may indicate that the evaluation of MSAs is more valuable for the recognition and management of IP patients than the diagnosis of IIMs [20,33,34]. According to the ERS/ATS statement, some of the MSA-IP patients who did not meet the criteria of IIMs can be diagnosed with IPAF [5]. However, compared with MSA-IP, the entity of IPAF is more heterogeneous, and the diagnosis of IPAF may lead to delayed clinical interventions [35]. Thus, we chose MSA-IP patients as the study population for cluster analysis.

In cluster 1, the UIP pattern on HRCT was observed in all patients. A previous study explored the clinical, radiological and histopathological features of the UIP pattern, which had been confirmed by surgical lung biopsies [36]. The results showed that various causes may lead to ILDs with UIP patterns. The most common disease are IPF, rheumatic ILD (RILD) and chronic hypersensitivity pneumonitis (CHP). The histopathological features of these diseases were different. Spatial and temporal heterogeneity, fibroblastic foci, a peripheral lobular distribution, and microscopic honeycomb were observed in IPF; airway-centred fibrosis, NSIP-like alveolar septal fibrosis, follicular bronchiolitis, and pleural fibrosis were observed in RILD. Finally, patchy fibrosis along the bronchovascular bundle with rare fibroblast foci, honeycomb cysts in the upper and lower lobes, extensive peribronchiolar metaplasia, and bridging fibrosis across lobules were observed in CHP [36]. The mean survival time of patients with RILD and a UIP was longer than that of patients with IPF and a UIP [37]. UIP patterns are diverse, and different histopathological features lead to differences in prognosis to some extent. Progressive-fibrosing ILDs (PF-ILDs) refer to fibrotic ILDs that present progressive phenotypes with multiple causes whose clinical, radiological and pathological characteristics overlap with IPF [38]. Given the similarities in the pathogenesis of fibrosis, the results of a clinical trial showed that Nintedanib can reduce the annual rate of decline in FVC in patients with PF-ILDs [39]. The UIP pattern is the representation of irreversible pulmonary fibrosis [40], and ILDs with the UIP pattern are in the category of PF-ILD [39]. In the present study, the MSA-IP patients of cluster 1 had a UIP pattern, which indicates that clinicians can differentiate the UIP pattern of possible MSA-IP, especially in cluster 1. In addition, antifibrotic medication may also be valuable for MSA-IP patients in cluster 1 [40].

All patients in cluster 2 were positive for anti-ARS antibodies, in which anti-Jo- 1 and anti-EJ antibodies were common, and were characterized by respiratory symptoms. Patients with anti-ARS antibodies often present myositis, IP, arthritis, mechanic's hands, Raynaud's phenomenon and fever, known as antisynthetase syndrome (ASS) [41,42]. In our study, cluster 2 patients were characterized by ASS and had 
more severe pulmonary involvement, such as a higher composite phhysiologic index, lower oxygenation index and diffuse GGO pattern. Research on ASS showed that the subtype of anti-ARS antibodies indicated certain clinical characteristics and predicated complications [41]. For example, ASS patients with anti-Jo-1, anti-EJ and anti-PL-7 antibodies were prone to myositis following ILDs [41]. However, the patients with various anti-ARS antibodies were not able to be distinguished by HRCT patterns of IPs. In cluster 3, anti-ARS antibodies were the main subtype of MSAs, but there were also anti-non-ARS MSAs. Patients in cluster 3 had multi-system involvement, but to a lesser degree. The NSIP pattern and acute onset were also features of cluster 3 . Compared with patients in cluster 3 , patients in cluster 2 had a shorter median survival time.

Cluster 4 patients had dyspnoea, decreased oxygenation and diffuse GGOs. Anti-MDA5 antibodies, acute onset and susceptibility to disease progression were representative features in cluster 4. Among DM patients in the U.S. and Japan, $13.1 \%$ to $37.3 \%$ were positive for anti-MDA5 antibodies [43,44]. Anti-MDA5 antibodies were found to be associated with rapidly progressive ILD and poor survival with a mortality rate as high as $71.4 \%$ [43]. The results of our study were consistent with previous studies. Initial triple therapy, including high-dose prednisolone, a calcineurin inhibitor and intravenous cyclophosphamide was more commonly used in survivors of rapidly progressive ILD with anti-MDA5 antibodies than in nonsurvivors, indicating that initial triple therapy may improve the prognosis of these patients [44]. For IP patients with anti-MDA5 antibodies who failed to respond to triple therapy, combined treatment with Tofacitinib might be beneficial in controlling disease progression [45]. Given the results of this study and previous studies, patients presenting with rapidly progressive IP should undergo autoantibody detection, particularly for anti-MDA5 antibodies, to increase diagnostic sensitivity [35].

Several limitations of this study should be considered. First, selection bias might exist because the enrolled patients did not fully represent the diversity of organ involvement in MSA-IP as they were derived from a single medical centre. Second, the clustering variables did not contain therapeutic data. In this study, MSA-IP patients were mainly treated with corticosteroids and immunosuppressive agents, including cyclophosphamide, methotrexate, azathioprine, mycophenolate mofetil, cyclosporine, or hydroxychloroquine, with or without antifibrotic therapy. Finally, the follow-up period was limited for observing disease progression in the patients. Prospective long-term longitudinal research is warranted to verify the four clusters of MSA-IP patients in this study.

\section{Conclusions}

We applied cluster analysis to MSA-IP for the first time, resulting in the categorization of four clusters. We hope that the results of this study will assist clinicians in evaluating the prognosis of patients with MSAIP when the symptoms are atypical and before the diagnosis of IIM and IP subtypes to assist treatment. However, the results of this study still need to be verified. Further studies are warranted to explore the correlation of clinical characteristics with underlying genetic mechanisms of corresponding MSA subtypes. 


\section{Abbreviations}

ACR, American College of Rheumatology; ADM, amyopathic dermatomyositis; anti-ARS, anti-aminoacyltRNA-synthetase antibodies; ASS, anti-synthetase syndrome; ATS, American Thoracic Society; CHP, chronic hypersensitivity pneumonitis; CTDs, connective tissue diseases; CTD-ILD, CTD-associated ILD; DLCO, diffusing capacity of the lung for carbon monoxide; DM, dermatomyositis; ERS, European Respiratory Society; EULAR, European League Against Rheumatism; FVC, forced vital capacity; GGO, ground glass opacity; HRCT, high-resolution computed tomography; Ig, immunoglobulin; IIM, idiopathic inflammatory myopathy; IIPs, idiopathic interstitial pneumonias; ILDs, Interstitial lung diseases; IP, interstitial pneumonia; IPAF, interstitial pneumonia with autoimmune features; IPF, idiopathic pulmonary fibrosis; MAA, myositis-associated autoantibody; MSA, myositis-specific autoantibody; MSA-IP, interstitial pneumonia patients with MSA; NSIP, nonspecific interstitial pneumonia; PF-ILDs, progressive-fibrosing ILDs; PM, polymyositis; RILD, rheumatic ILD; UIP, usual interstitial pneumonia.

\section{Declarations}

\section{Ethics approval and consent to participate}

The protocol of the prospective study was approved by the Institutional Review Board of Beijing ChaoYang Hospital. Written informed consents were obtained from all patients.

\section{Consent for publication}

Not applicable.

\section{Availability of data and material}

The datasets used in the current study are available from the corresponding author upon reasonable request.

\section{Competing interests}

The authors have no conflicts of interest to declare.

\section{Funding}


National Natural Science Foundation of China (81970061) only financially supported this study, and didn't take any part in the design of the study, and collection, analysis, and interpretation of data and in writing this manuscript.

\section{Authors' contributions}

YHL was responsible for completing the analysis of data and writing. SWY and YLF performed all data collection. NW and YRW were responsible for recruiting the patients and collecting plasma samples. QY contributed as primary investigator and was responsible for designing the study, recruiting the patients and writing the manuscript. All authors have read and approved the final manuscript.

\section{Acknowledgements}

We thank all patients and investigators who were involved in this study. We express our thanks to Miss Moyang Xu of University of Michigan-Ann Arbor for polishing language and grammar of the manuscript.

\section{References}

1. Antoniou K M, Margaritopoulos G A, Tomassetti S, Bonella F, Costabel U, Poletti V. Interstitial lung disease. Eur Respir Rev. 2014;23:40-54.

2. Society A T, Society E R. American Thoracic Society/European Respiratory Society International Multidisciplinary Consensus Classification of the Idiopathic Interstitial Pneumonias. This joint statement of the American Thoracic Society (ATS), and the European Respiratory Society (ERS) was adopted by the ATS board of directors, June 2001 and by the ERS Executive Committee, June 2001. Am J Respir Crit Care Med. 2002;165:277-304.

3. Ryerson C J, Collard H R. Update on the diagnosis and classification of ILD. Curr. Opin. Pulm. Med. 2013;19:453-459.

4. Vij R, Strek M E. Diagnosis and treatment of connective tissue disease-associated interstitial lung disease. Chest. 2013;143:814-824.

5. Fischer A, Antoniou K M, Brown K K, Cadranel J, Corte T J, du Bois R M, et al. An official European Respiratory Society/American Thoracic Society research statement: Interstitial pneumonia with autoimmune features. Eur. Respir. J. 2015;46:976-987.

6. Koo S M, Uh S T. Treatment of connective tissue disease-associated interstitial lung disease: The pulmonologist's point of view. Korean J. Intern. Med. 2017;32:600-610.

7. Fischer A, West S G, Swigris J J, Brown K K, du Bois R M. Connective tissue disease-associated interstitial lung disease: A call for clarification. Chest. 2010;138:251-256. 
8. Le Goff B, Cherin P, Cantagrel A, Gayraud M, Hachulla E, Laborde F, et al. Pneumomediastinum in interstitial lung disease associated with dermatomyositis and polymyositis. Arthritis Rheum. 2009;61:108-118.

9. Bohan A, Peter J B. Polymyositis and dermatomyositis (second of two parts). N Engl J Med. 1975;292:403-407.

10. Morisset J, Johnson C, Rich E, Collard H R, Lee J S. Management of Myositis-Related interstitial lung disease. Chest. 2016;150:1118-1128.

11. Labirua A, Lundberg I E. Interstitial lung disease and idiopathic inflammatory myopathies: Progress and pitfalls. Curr. Opin. Rheumatol. 2010;22:633-638.

12. Mimori T, Nakashima R, Hosono Y. Interstitial lung disease in myositis: Clinical subsets, biomarkers, and treatment. Curr Rheumatol Rep. 2012;14:264-274.

13. Fathi M, Lundberg I E. Interstitial lung disease in polymyositis and dermatomyositis. Curr. Opin. Rheumatol. 2005;17:701-706.

14. Fathi M, Vikgren J, Boijsen M, Tylen U, Jorfeldt L, Tornling G, et al. Interstitial lung disease in polymyositis and dermatomyositis: Longitudinal evaluation by pulmonary function and radiology. Arthritis Rheum. 2008;59:677-685.

15. Marie I, Hachulla $E$, Cherin $P$, Dominique $S$, Hatron $P Y$, Hellot $M F$, et al. Interstitial lung disease in polymyositis and dermatomyositis. Arthritis Rheum. 2002;47:614-622.

16. Chen I J, Jan W Y, Lin C W, Fan K W, Luo S F, Ho H H, et al. Interstitial lung disease in polymyositis and dermatomyositis. Clin. Rheumatol. 2009;28:639-646.

17. Richards T J, Eggebeen A, Gibson K, Yousem S, Fuhrman C, Gochuico B R, et al. Characterization and peripheral blood biomarker assessment of anti-Jo-1 antibody-positive interstitial lung disease. Arthritis Rheum. 2009;60:2183-2192.

18. Tieu J, Lundberg I E, Limaye V. Idiopathic inflammatory myositis. Best Pract Res Clin Rheumatol. 2016;30:149-168.

19. Ghirardello A, Zampieri S, Tarricone E, laccarino L, Bendo R, Briani C, et al. Clinical implications of autoantibody screening in patients with autoimmune myositis. Autoimmunity. 2006;39:217-221.

20. Tanizawa K, Handa T, Nakashima R, Kubo T, Hosono Y, Watanabe K, et al. The long-term outcome of interstitial lung disease with anti-aminoacyl-tRNA synthetase antibodies. Respir Med. 2017;127:5764.

21. van de Velden M, D'Enza A I, Palumbo F. Cluster correspondence analysis. Psychometrika. 2017;82:158-185.

22. Szodoray P, Hajas A, Kardos L, Dezso B, Soos G, Zold E, et al. Distinct phenotypes in mixed connective tissue disease: Subgroups and survival. Lupus. 2012;21:1412-1422.

23. Travis W D, Costabel U, Hansell D M, King T J, Lynch D A, Nicholson A G, et al. An official American Thoracic Society/European Respiratory Society statement: Update of the international 
multidisciplinary classification of the idiopathic interstitial pneumonias. Am J Respir Crit Care Med. 2013;188:733-748.

24. Lundberg I E, Tjarnlund A, Bottai M, Werth V P, Pilkington C, Visser M, et al. 2017 European League Against Rheumatism/American College of Rheumatology classification criteria for adult and juvenile idiopathic inflammatory myopathies and their major subgroups. Ann. Rheum. Dis. 2017;76:19551964.

25. Targoff I N. Laboratory testing in the diagnosis and management of idiopathic inflammatory myopathies. Rheum Dis Clin North Am. 2002;28:859-890.

26. Targoff I N. Myositis specific autoantibodies. Curr Rheumatol Rep. 2006;8:196-203.

27. Nakashima R, Imura Y, Kobayashi S, Yukawa N, Yoshifuji H, Nojima T, et al. The RIG-I-like receptor IFIH1/MDA5 is a dermatomyositis-specific autoantigen identified by the anti-CADM-140 antibody. Rheumatology (Oxford). 2010;49:433-440.

28. Wanger J, Clausen J L, Coates A, Pedersen O F, Brusasco V, Burgos F, et al. Standardisation of the measurement of lung volumes. Eur. Respir. J. 2005;26:511-522.

29. Zhu H, Wu C, Jiang N, Wang Y, Zhao J, Xu D, et al. Identification of 6 dermatomyositis subgroups using principal component analysis-based cluster analysis. Int J. Rheum Dis. 2019;22:1383-1392.

30. Galie N, Hoeper M M, Humbert M, Torbicki A, Vachiery J L, Barbera J A, et al. Guidelines for the diagnosis and treatment of pulmonary hypertension. Eur. Respir. J. 2009;34:1219-1263.

31. Ryerson C J, Abbritti M, Ley B, Elicker B M, Jones K D, Collard H R. Cough predicts prognosis in idiopathic pulmonary fibrosis. Respirology. 2011;16:969-975.

32. Fidler L, Doubelt I, Kandel S, Fisher J H, Mittoo S, Shapera S. Screening for myositis antibodies in idiopathic interstitial lung disease. Lung. 2019;197:277-284.

33. Hervier B, Devilliers H, Stanciu R, Meyer A, Uzunhan Y, Masseau A, et al. Hierarchical cluster and survival analyses of antisynthetase syndrome: Phenotype and outcome are correlated with anti-tRNA synthetase antibody specificity. Autoimmun. Rev. 2012;12:210-217.

34. Mariampillai K, Granger B, Amelin D, Guiguet M, Hachulla E, Maurier F, et al. Development of a new classification system for idiopathic inflammatory myopathies based on clinical manifestations and Myositis-Specific autoantibodies. Jama Neurol. 2018;75:1528-1537.

35. Jablonski R, Bhorade S, Strek M E, Dematte J. Recognition and management of myositis-associated rapidly progressive interstitial lung disease. Chest. 2020;

36. Smith M, Dalurzo M, Panse P, Parish J, Leslie K. Usual interstitial pneumonia-pattern fibrosis in surgical lung biopsies. Clinical, radiological and histopathological clues to aetiology. J. Clin. Pathol. 2013;66:896-903.

37. Park J H, Kim D S, Park I N, Jang S J, Kitaichi M, Nicholson A G, et al. Prognosis of fibrotic interstitial pneumonia: Idiopathic versus collagen vascular disease-related subtypes. Am J Respir Crit Care Med. 2007;175:705-711. 
38. Cottin V, Hirani N A, Hotchkin D L, Nambiar A M, Ogura T, Otaola M, et al. Presentation, diagnosis and clinical course of the spectrum of progressive-fibrosing interstitial lung diseases. Eur Respir Rev. 2018;27:

39. Flaherty K R, Brown K K, Wells A U, Clerisme-Beaty E, Collard H R, Cottin V, et al. Design of the PF-ILD trial: A double-blind, randomised, placebo-controlled phase III trial of nintedanib in patients with progressive fibrosing interstitial lung disease. BMJ Open Respir Res. 2017;4:e212.

40. Raghu G. Idiopathic pulmonary fibrosis: Shifting the concept to irreversible pulmonary fibrosis of many entities. Lancet Respir Med. 2019;7:926-929.

41. Hamaguchi Y, Fujimoto M, Matsushita T, Kaji K, Komura K, Hasegawa M, et al. Common and distinct clinical features in adult patients with anti-aminoacyl-tRNA synthetase antibodies: Heterogeneity within the syndrome. Plos One. 2013;8:e60442.

42. Watanabe K, Handa T, Tanizawa K, Hosono Y, Taguchi Y, Noma S, et al. Detection of antisynthetase syndrome in patients with idiopathic interstitial pneumonias. Respir Med. 2011;105:1238-1247.

43. Moghadam-Kia S, Oddis C V, Sato S, Kuwana M, Aggarwal R. Anti-Melanoma DifferentiationAssociated gene 5 is associated with rapidly progressive lung disease and poor survival in US patients with amyopathic and myopathic dermatomyositis. Arthritis Care Res (Hoboken). 2016;68:689-694.

44. Motegi S I, Sekiguchi A, Toki S, Kishi C, Endo Y, Yasuda M, et al. Clinical features and poor prognostic factors of anti-melanoma differentiation-associated gene 5 antibody-positive dermatomyositis with rapid progressive interstitial lung disease. Eur. J. Dermatol. 2019;29:511-517.

45. Kurasawa K, Arai S, Namiki Y, Tanaka A, Takamura Y, Owada T, et al. Tofacitinib for refractory interstitial lung diseases in anti-melanoma differentiation-associated 5 gene antibody-positive dermatomyositis. Rheumatology (Oxford). 2018;57:2114-2119.

\section{Tables}

Table 1 Clinical characteristics of four clusters

\begin{tabular}{cccccccc}
\hline & All & Cluster 1 & Cluster 2 & Cluster 3 & Cluster 4 & $\mathrm{X}^{2}$ & $P^{*}$ value \\
\hline Fever, n (\%) & $46(37.1 \%)$ & $5(20.0 \%)$ & $20(37.0 \%)$ & $4(26.7 \%)$ & $17(56.7 \%)$ & 8.755 & 0.033 \\
Proximal muscle weakness, n (\%) & $10(8.1 \%)$ & 0 & 0 & $10(66.7 \%)$ & 0 & $43.232<0.001$ \\
\hline Dysphagia, n (\%) & $4(3.2 \%)$ & $1(4.0 \%)$ & $1(1.9 \%)$ & $2(13.3 \%)$ & 0 & 6.283 & 0.099 \\
\hline Cough, n (\%) & $103(83.1 \%)$ & $22(88.0 \%)$ & $48(88.9 \%)$ & $6(40.0 \%)$ & $28(93.3 \%)$ & $22.194<0.001$ \\
\hline Dyspnea, n (\%) & $95(76.6 \%)$ & $17(68.0 \%)$ & $48(88.9 \%)$ & $6(40.0 \%)$ & $24(80.0 \%)$ & 15.546 & 0.001 \\
\hline Arthralgia, n (\%) & $32(25.8 \%)$ & $3(12.0 \%)$ & $14(25.9 \%)$ & $7(46.7 \%)$ & $8(26.7 \%)$ & 5.750 & 0.125 \\
\hline Morning stiffness, n (\%) & $11(8.9 \%)$ & 0 & $4(7.4 \%)$ & $4(26.7 \%)$ & $3(10.0 \%)$ & 7.279 & 0.036 \\
\hline Joint swelling, n (\%) & $11(8.9 \%)$ & $1(4 \%)$ & $4(7.4 \%)$ & $6(40 \%)$ & 0 & 14.861 & 0.001 \\
\hline
\end{tabular}

Values were given as $\mathrm{n}(\%)$. 
*, comparisons among four clusters.

Table 2 MSA and parameters of four clusters

\begin{tabular}{|c|c|c|c|c|c|c|c|}
\hline & All & Cluster 1 & Cluster 2 & Cluster 3 & Cluster 4 & $\mathrm{~T} / \mathrm{U} / \mathrm{X}^{2}$ & $\begin{array}{c}P^{*} \\
\text { value } \\
\end{array}$ \\
\hline Anti-ARS, n (\%) & $75(60.5 \%)$ & $11(44.0 \%)$ & $54(100 \%)$ & $10(66.7 \%)$ & 0 & 84.280 & $<0.001$ \\
\hline $\begin{array}{l}\text { Anti-non-ARS } \\
\text { MSA, n (\%) }\end{array}$ & 49 (39.5\%) & $14(56.0 \%)$ & 0 & $5(33.3 \%)$ & $30(100 \%)$ & 84.280 & $<0.001$ \\
\hline Anti-Jo-1, n (\%) & $20(16.1 \%)$ & $4(16.0 \%)$ & $13(24.1 \%)$ & $3(20 \%)$ & 0 & 10.293 & 0.012 \\
\hline Anti-PL-7, n (\%) & $20(16.1 \%)$ & $4(16.0 \%)$ & $12(22.2 \%)$ & $4(26.7 \%)$ & 0 & 10.441 & 0.010 \\
\hline Anti-PL-12, n (\%) & $9(7.3 \%)$ & $2(8.0 \%)$ & 7 (13.0\%) & 0 & 0 & 5.260 & 0.110 \\
\hline Anti-OJ, n (\%) & $7(5.7 \%)$ & $1(4.0 \%)$ & $5(9.3 \%)$ & $1(6.7 \%)$ & 0 & 3.088 & 0.326 \\
\hline Anti-EJ, n (\%) & $19(15.3 \%)$ & 0 & $17(31.5 \%)$ & $2(13.3 \%)$ & 0 & 19.819 & $<0.001$ \\
\hline Anti-SRP, n (\%) & $8(6.5 \%)$ & $4(16.0 \%)$ & 0 & $1(6.7 \%)$ & $3(10 \%)$ & 5.695 & 0.092 \\
\hline Anti-Mi-2 $\alpha, \mathrm{n}(\%)$ & $3(2.4 \%)$ & $2(8.0 \%)$ & 0 & 0 & $1(3.3 \%)$ & 2.310 & 0.421 \\
\hline Anti-Mi-2ß, n (\%) & $10(8.3 \%)$ & $2(8.0 \%)$ & 0 & 0 & $8(26.7 \%)$ & 16.133 & $<0.001$ \\
\hline Anti-TIF1 $\gamma, \mathrm{n}(\%)$ & $8(6.5 \%)$ & $3(12.0 \%)$ & 0 & 0 & $5(16.7 \%)$ & 4.211 & 0.211 \\
\hline Anti-MDA5, n (\%) & $15(11.8 \%)$ & $2(8.0 \%)$ & 0 & $2(13.3 \%)$ & $11(36.7 \%)$ & 22.219 & $<0.001$ \\
\hline Anti-NXP2, n (\%) & $4(3.2 \%)$ & 0 & 0 & $2(13.3 \%)$ & $2(6.7 \%)$ & 5.759 & 0.076 \\
\hline Anti-SAE1, n (\%) & $1(0.8 \%)$ & $1(4.0 \%)$ & 0 & 0 & 0 & 1.823 & 0.671 \\
\hline RF positive, n (\%) & $13(10.5 \%)$ & 0 & $4(8.9 \%)$ & $3(25.0 \%)$ & $6(26.1 \%)$ & 8.290 & 0.024 \\
\hline cTNI, ng/ml & $0(0,0.01)$ & $0(0,0.01)$ & $0(0,0.01)$ & $\begin{array}{c}0.03(0.02 \\
0.06)\end{array}$ & $0.01(0,0.01)$ & 9.904 & 0.019 \\
\hline OI, mmHg & $\begin{array}{c}353.7(311.9 \\
434.2)\end{array}$ & $\begin{array}{c}356.0(337.9 \\
397.1)\end{array}$ & $\begin{array}{c}353.7(296.0 \\
419.1)\end{array}$ & $\begin{array}{c}436.8(325.1 \\
457.8)\end{array}$ & $\begin{array}{c}345.6(279.3 \\
410.5)\end{array}$ & 1.867 & 0.600 \\
\hline CPI & $38.9 \pm 14.3$ & $34.6 \pm 13.9$ & $43.2 \pm 12.2$ & $30.5 \pm 18.9$ & $40.3 \pm 11.4$ & 3.314 & 0.025 \\
\hline
\end{tabular}

Values were given as $\mathrm{n}(\%)$, median (interquartile range) or mean (standard deviation).

*, comparisons among four clusters.

Abbreviations: RF, rheumatoid factor; cTNI, cardiac troponin I; CPI, composite physiologic index; OI, oxygenation index; ARS, aminoacyl-tRNA synthetase; MSA, myositis specific antibodies.

Table 3 Multivariable Logistic regression model for acute onset 


\begin{tabular}{cccc}
\hline & $P$ value & OR & $95 \%$ CI of OR \\
\hline Age $^{*}$ & 0.127 & 1.028 & $0.992-1.066$ \\
Female & 0.587 & 1.364 & $0.446-4.175$ \\
\hline Non-smokers $^{\#}$ & 0.643 & & \\
\hline Ex-smokers $^{2}$ & 0.618 & 1.406 & $0.369-5.365$ \\
\hline Current smokers & 0.648 & 0.732 & $0.192-2.795$ \\
\hline Cluster\& & 0.022 & \\
\hline Cluster 2 & 0.074 & 2.883 & $0.903-9.204$ \\
\hline Cluster 3 & 0.011 & 6.682 & $1.560-28.622$ \\
\hline Cluster 4 & 0.005 & 6.057 & $1.715-21.388$ \\
\hline
\end{tabular}

${ }^{*}$, age at the diagnosis; ${ }^{*}$, take non-smokers as a reference; ${ }^{\&}$, take cluster1 as a reference.

Abbreviations: OR, odds ratio

Table 4 Multivariable Cox proportional hazards model for interstitial pneumonia progression or death

\begin{tabular}{cccc}
\hline Variables & $P$ value & HR & $95 \%$ CI of HR \\
\hline Female & 0.683 & 0.839 & $0.362-1.946$ \\
Age $^{*}$ & 0.502 & 1.009 & $0.983-1.036$ \\
\hline Non-smokers $^{\#}$ & 0.524 & & \\
\hline Current smokers $^{2}$ & 0.337 & 0.585 & $0.196-1.749$ \\
\hline Ex-smokers & 0.310 & 0.593 & $0.217-1.626$ \\
\hline Cluster & 0.035 & & \\
\hline Cluster 2 & 0.367 & 1.436 & $0.654-3.152$ \\
\hline Cluster 3 & 0.291 & 0.514 & $0.150-1.767$ \\
\hline Cluster 4 & 0.026 & 2.711 & $1.128-6.519$ \\
\hline
\end{tabular}

${ }^{*}$, age at the diagnosis; ${ }^{*}$, take non-smokers as a reference; ${ }^{\&}$, take cluster1 as a reference.

Abbreviations: HR, hazard ratio.

\section{Figures}




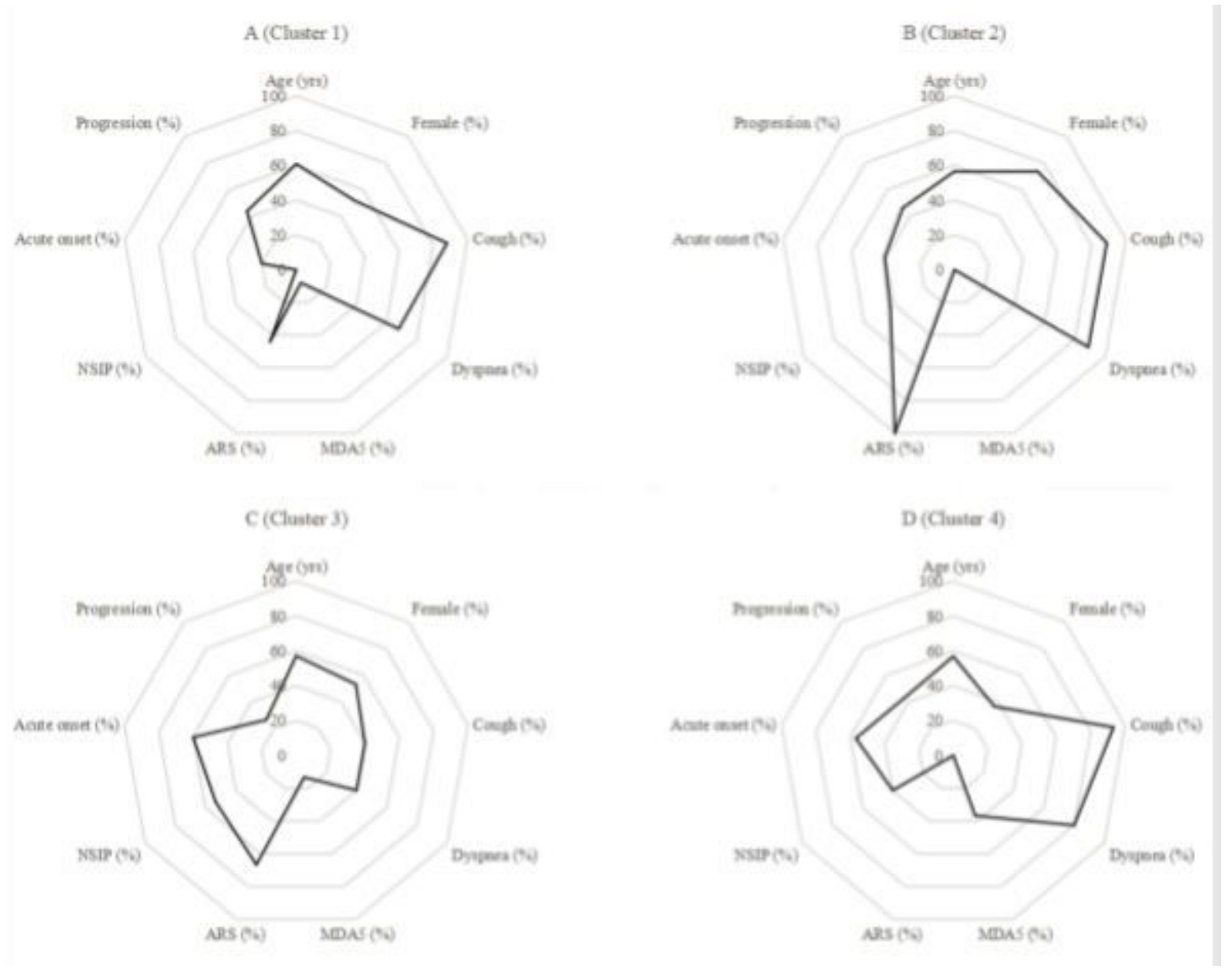

Figure 1

Radar plot depicting the distribution of demographics, clinical characteristics, MSA subtypes and HRCT findings among four clusters. \%, the percentage of patients with corresponding characteristics; yrs, age at diagnosis. 


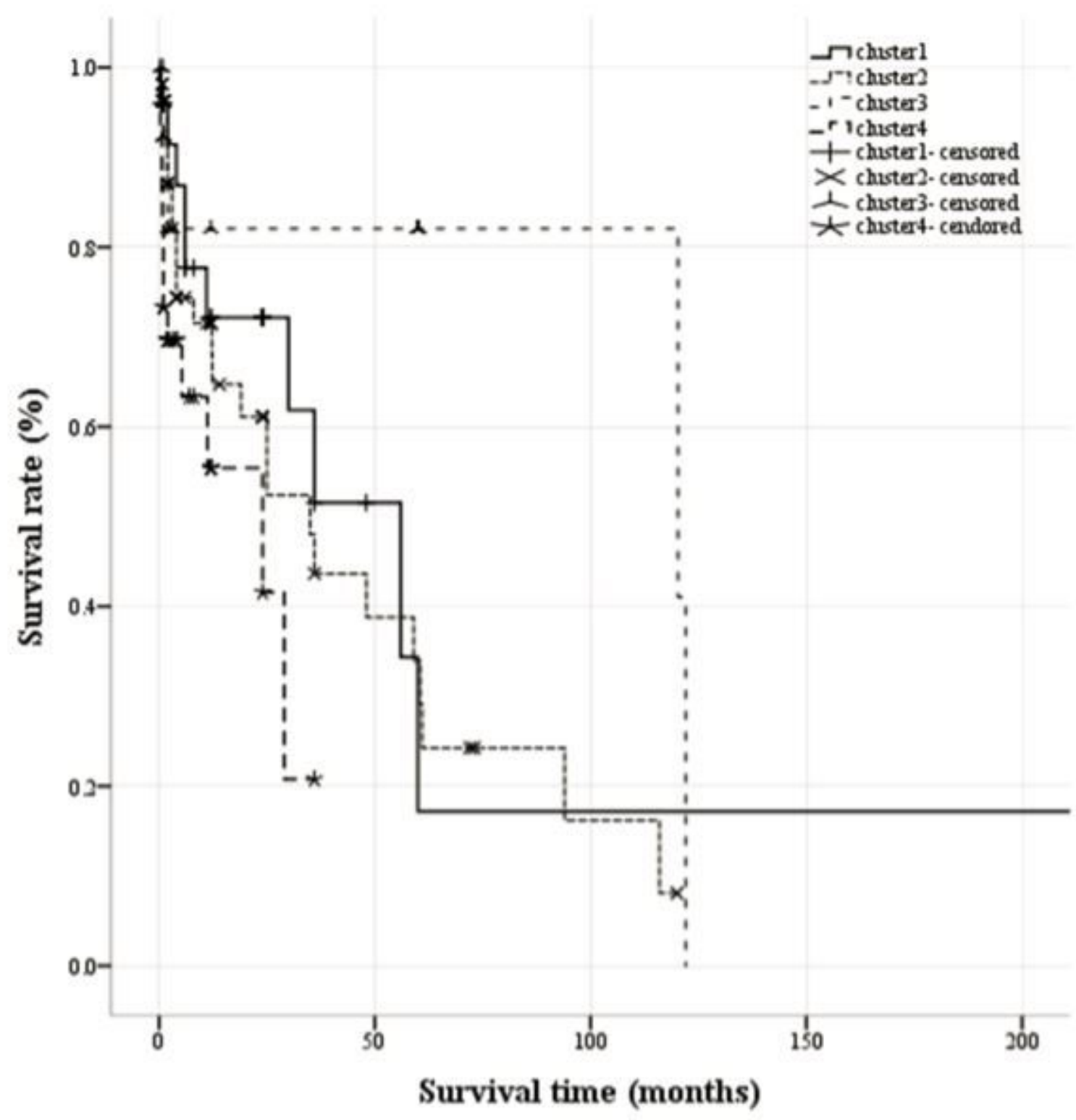

Figure 2

Kaplan-Meier curves of MSA-IP patients in four clusters (cluster 1, solid line; cluster 2, dotted line; cluster 3 , short dashed line; cluster 4, long dashed line). Survival time was calculated from the onset of IP-related symptoms to outcomes or the end of the follow-up period. The median survival time of all patients was 36 months (range, 0.1-360 months). The median survival time of cluster 4 was 24 months, which was the

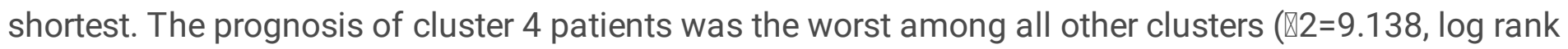
$\mathrm{P}=0.028)$.

\section{Supplementary Files}

This is a list of supplementary files associated with this preprint. Click to download.

- Additionalfile5.docx 
- Additionalfile4.docx

- Additionalfile3.docx

- Additionalfile2.docx

- Additionalfile1.pdf

- RevisedAdditionalfile6.docx 